\title{
¿Sabemos cuáles son los efectos adversos de los medicamentos que recetamos?
}

\author{
I. Sánchez Lite, A. de la Cal de la Fuente*, R. Frutos Llanes, \\ M. CANO DEL POZO** \\ Médico Residente de Medicina Familiar y Comunitaria. *Médico Especialista \\ en MFyC**Médico Residente de Neurofisiología Clínica. \\ Centro de Salud Casa del Barco. Área Oeste. Valladolid
}

\section{RESUMEN}

Muchos de los medicamentos que utilizamos en nuestras consultas, y a los que presuponemos ino cuidad, pueden producir multitud de efectos secun darios que en ocasiones pueden llegar a ser letales. La agranulocitosis es una poco frecuente alteración sanguínea consistente en una neutropenia severa que asocia infecciones graves y una elevada morta lidad. Un gran número de medicamentos se han asociado con la producción de agranulocitosis anti bióticos, antiinflamatorios , antidepresivos, etc... Dentro de estos medicamentos el metamizol ha sido uno de los fármacos que clásicamente se ha asocia do a este trastorno sanguineo. Ampliamente utiliza do, el metamizol, goza de gran popularidad no sólo entre el ambiente médico sino entre los mismos pacientes que pueden conseguirlo sin receta médica en cualquier farmacia dentro de nuestro país. Aun que la asociación de agranulocitosis y metamizol es poco frecuente debemos tenerla presente por las graves consecuencias que puede traer. Tal fue el caso de la paciente que describimos, la cual tras haber recibido metamizol debió ser atendida urgente mente en su domicilio, siendo posteriormente remitida al hospital. La analítica en urgencias mos tró una agranulocitosis. Posteriormente ingresaría en la unidad de cuidados intensivos donde, a pesar de las medidas de soporte y la antibioterapia, la paciente falleció a las pocas horas.

Palabras clave: Agranulocitosis. Antinflamato rias ni esteroideos. Dipirona. Neutropenia. Efectos adversos. Mortalidad.
Are we aware of the side effects of the drugs we prescribe?

\section{ABSTRACT}

Many of the medicines used daily and which are supposed harmless, may in fact produce numerous side-effects which can, on occasions, be life-threa tening. Agranulocytosis is an uncommon alteration of the blood consisting of severe neutropenia with serious associated infections and a high mortality rate. A great number of medications have been as sociated with the production of agranulocytosis: antibiotics, anti-inflammatory, anti-depressants, etc. Of these medicines metamizol has been one of the most closely-associated with this blood disor der. Widely used, metamizol, is very popular not only in medical circles, but also amongst patients who are able to buy it without prescription from any pharmacy in our country. Although the asso ciation between agranulocytosis and metamizol is not common, we should be aware of the grave consequences which may arise. This was the case of the patient described who, having taken metamizol, required urgent treatment at home, before being admitted to hospital. Emergency room analysis re vealed agranulocitosis. The patient was admitted to the Intensive Care Unit where, in spite of sup port and anti-biotherapy, she died within a few hours.

Key words: Agranulocytosis. Anti-inflammatory agents. Non-steroidal. Dipyrone. Neutropenia. Side Effects. Mortality.

Aceptación: 17-01-02 


\section{INTRODUCCIÓN}

Los trastornos hematológicos causados por la utilización de diversos fármacos son reacciones adversas que, aún siendo frecuentes en Atención Primaria, se diagnostican en pocas ocasiones.

Dentro de estos trastornos hematológicos se encuentra la agranulocitosis, caracterizada por una neutropenia severa, menos de $10^{\circ}$ granulocitos/litro, reversible, acompañada de infecciones agudas y elevada gravedad.

El síndrome clínico fue descrito por Shultz en 1922, posteriormente en el año 1935 Blake publica el primer caso en el que se implica el metamizol con la agranulocitosis.

Presentamos el caso de una paciente que recibió metamizol por un episodio de lumbalgia aguda.

\section{CASO CLÍNICO}

Paciente mujer de 53 años de edad que, de forma esporádica, toma metamizol por diferentes causas, la última de las cuales fue en diciembre de 2000 por dolores generalizados. En febrero de 2001, debido a un episodio de lumbalgia aguda, vuelve a recibir metamizol a una dosis de $575 \mathrm{mg}$ cada 8 horas durante 6 días. A los 6 días de finalizar el tratamiento fue requerida nuestra presencia, de forma urgente, en su domicilio por un cuadro consistente en hemoptisis, dolor en el pecho, erupción cutánea, dificultad respiratoria y fiebre. El día anterior había presentado malestar general, anorexia y un cuadro seudogripal.

Como antecedentes personales presentaba síndrome de ansiedad en tratamiento con lorazepam y paroxetina dede hacía 2 años, histerectomía y doble anexectomía en febrero de 1997, en tratamiento con tibolona y calcio. Durante la exploración la paciente estaba agitada, con gran trabajo respiratorio, una TA de 88/56 y una frecuencia cardiaca de 70 latidos por minuto. Presentaba cianosis y una erupción máculo-papulosa en cara, cuello, tronco, brazos y muslos. En la auscultación cardiopulmonar encontramos una taquicardia rítmica con hipoventilación global. El abdomen estaba blando, depresible sin masas, megalias ni signos de irritación peritoneal. En la exploración neurológica no se encontraron alteraciones y las extremidades estaban mal perfundidas, sin edemas ni signos de flebitis.

La paciente fue remitida, de forma urgente, al hospital donde posteriormente ingresaría en la UCI. A su llegada a urgencias la analítica fue la siguiente: Sistemático de sangre: 500 leucocitos $/ \mu \mathrm{L}$ (N 1,4\%, L 92,2\%). Se realizó biopsia de médula ósea objetivándose hipoplasia relativa de tejido hematopoyético, sin alteraciones morfológicas, serie roja y plaquetas normales. Gasometría arterial ba- sal: pO2 37, pCO2 51, pH 7.326, HCO3 27. Bioquímica: glucosa $188 \mathrm{mg} / \mathrm{dL}$, resto normal incluídos enzimas cardiacos. Coagulación: tiempo de protrombina $18,6 \mathrm{seg}$, actividad de protrombina $36 \%$, INR 2,05, tiempo de tromboplastina parcial activada 29,4 seg, fibrinógeno $800 \mathrm{mg} \%$. Tinción de Gram del esputo observándose numerosos cocos Gram + y hematíes. En el cultivo del esputo y el hemocultivo se aisló Stafilococo Aureus. El urocultivo, la detección de antígenos en orina de Legionella y Streptococo, la baciloscopia y tinción de Ziehl-Neelsen en esputo, el cultivo de hongos en esputo y la serología para virus en sangre fueron negativos.

En la radiografía de tórax se apreciaba un infiltrado alveolo-intersticial basal bilateral y una zona de condensación alveolar en lóbulo superior derecho. El electrocardiograma mostraba un ritmo sinusal a $140 \mathrm{lpm}$, eje a $+60^{\circ}$, sin alteraciones de la conducción ni de la repolarización.

Se realizaron otra serie de exploraciones complementarias: Gastroscopia: no se evidenciaron zonas de sangrado digestivo. Nasofibroscopia: pequeños puntos hemáticos en cavum; hipofaringe y laringe normales. Broncoscopia: sangre en árbol bronquial, sin apreciarse punto sangrante en tráquea, bronquios principales ni lobares. TAC torácico: condensaciones pulmonares bilaterales difusas, con imagen de broncograma aéreo; opacificación completa de lóbulo inferior derecho, lóbulo inferior izquierdo, lóbulo medio derecho; distribución parcheada en LSD y língula; bronquios principales, lobares y segmentarios permeables.

Durante su ingreso en la UCI fue necesaria la intubación y se comenzó tratamiento con antibioterapia y medidas de soporte. La paciente presentó un cuadro de shock refractario con progresión a fallo multiorgánico falleciendo el mismo día de su ingreso.

Se efectuó necropsia llegando a los siguientes diagnósticos: edema pulmonar masivo bilateral con extensas áreas de hemorragia y presencia de abundantes colonias bacterianas de localización intravascular y alveolar. Derrame pleural. Hipoplasia relativa de médula ósea. Hígado de éstasis. Congestión vascular generalizada.

\section{DISCUSIÓN}

Los fármacos suponen la primera causa de agranulocitosis en nuestro medio ${ }^{1,2}$. La agranulocitosis inducida por fármacos es consecuencia de una reacción idiosincrásica, por tanto realmente infrecuente. En el caso del metamizol el riesgo de agranulocitosis es inferior a un caso por millón de tandas de tratamiento ${ }^{3}$.

Multitud de fármacos se han asociado con la producción de agranulocitosis: antihistamínicos, 
antidiabéticos orales, antiinflamatorios, neurolépticos, antihipertensivos, ansiolíticos, antidepresivos, antibióticos y otros.

En el Estudio Internacional sobre la Agranulocitosis y la Anemia Aplásica, realizado en áreas metropolitanas de Barcelona, Milán, Ulm, Budapest, Sofía, Estocolmo y Uppsala la incidencia de agranulocitosis inducida por fármacos oscila entre 1,7 casos por millón de habitantes y año en Milán y los 9 casos por millón de habitantes y año en Budapest. En el área de Barcelona se observó una incidencia de 5,7 casos por millón de habitantes y año ${ }^{3}$. En dicho estudio se encontraron diferencias significativas entre las diferentes regiones en cuanto al riesgo de presentar agranulocitosis relacionada con la utilización de metamizol. La causa de esta variabilidad regional no está aclarada. Se ha intentado explicar por factores derivados del propio estudio, como sesgos no detectados o defectos metodológicos, factores del propio paciente, factores ambientales, o incluso, a infecciones virales. En este mismo estudio se observó que el $4 \%$ de los pacientes que sufrieron agranulocitosis en comparación con el $0,5 \%$ de los controles, tenían antecedentes de mononucleosis. Un intento de explicación de este hallazgo sería que el aumento del riesgo de padecer agranulocitosis estaría ligado a la presencia del virus de Epstein Barr en el genoma celular'.

El pronóstico de este trastorno hematológico ha ido variando a lo largo del tiempo, mejorando gracias a la aparición de los antibióticos, aún así asocia una alta mortalidad, oscilando entre el 16 y el $30 \%{ }^{5}$. El pronóstico depende sobre todo de factores como la duración y la gravedad del trastorno, que aumentan el riesgo de infecciones, aunque otros como la edad, la rapidez de instauración del tratamiento antibiótico, la insuficiencia renal y la bacteriemia van a jugar un papel importante ${ }^{6}$. En el caso de nuestra paciente sólo encontramos como factor de riesgo la bacteriemia objetivada mediante hemocultivo. La clínica fugaz y la evolución fatal nos hacen pensar en la posible asociación de distintos mecanismos causales que pudieran actuar variando el pronóstico. Factores genéticos, como ciertos alelos del HLA, el HLA-DQwl, y alteraciones cromosómicas, se han objetivado en pacientes que han sufrido agranulocitosis por metamizol ${ }^{7}$.

Se han encontrado varios mecanismos patogénicos, hematotoxicidad directa sobre células periféricas, hematotoxicidad hacia células madres sensibles e hipersensibilidad mediada por anticuerpos. Este último requeriría una exposición previa del paciente y es el mecanismo de actuación en el que se ha implicado al metamizol ${ }^{8}$.

Los pacientes presentan infecciones agudas (septicemia por neumonía, lesiones orofaríngeas o anorectales), fiebre elevada, escalofríos, cefalea, dolor de garganta y otros síntomas acompañados de neutropenia severa ${ }^{8}$.
El antecedente de exposición al fármaco, la clínica, el estudio de médula ósea y la resolución del cuadro con la retirada del medicamento nos ayudan a llegar al diagnóstico. El estudio de la médula ósea muestra una disminución de las células granulopoyéticas con una importante reducción de las formas maduras e hiperplasia de las células precursoras, en cambio los sistemas eritroblástico y megacariocítico no presentan alteraciones ${ }^{3,8,6}$. En nuestra paciente no se pudo objetivar la hiperplasia de células precursoras, quizás debido a la rapidez de instauración del cuadro y al fatal desenlace. El informe anatomopatológico hacía referencia a una hipoplasia relativa de tejido hematopoyético que podría explicarse por la concurrencia de varios factores, por una parte la agresividad del agente infeccioso y por otra la falta de reacción del propio paciente por causas idiopáticas o también como consecuencia de toxicidad hematológica. En este sentido la agranulocitosis es la expresión más frecuente descrita de toxicidad hematológica asociada a la ingesta de fármacos.

El intervalo de tiempo entre la administración del fármaco y la aparición de la agranulocitosis se relaciona, de forma directa, con la inmadurez de la célula lesionada y el tipo de inductor, así el metamizol produce, en pocas horas, un descenso de leucocitos y, en pocos días, la aparición de signos de infección ${ }^{8}$. Nuestra paciente, como hemos comentado, cumplimentó seis días de tratamiento, la clínica comenzó a los trece días de inicio del mismo y fue objetivada una infección por Stafilococo aureus, lo cual concuerda con la secuencia temporal descrita. En general el recuento de neutrófilos se normaliza a las 2-4 semanas de retirada del fármaco causal ${ }^{8}$.

El pilar del tratamiento radica en la suspensión del agente responsable junto con medidas de soporte (aislamiento, antibioterapia, sueroterapia, etc...). La utilización de factores de crecimiento hematopoyéticos, extendida en los últimos años, parece acortar la duración del cuadro ${ }^{5}$ y disminuir la mortalidad $^{9,10}$, aunque en otros casos su uso no reportó diferencias en cuanto a esta última ${ }^{1}$.

El metamizol es un fármaco retirado en países como los EE.UU., el Reino Unido y los países nórdicos. En EE.UU. fue retirado a principios de los años setenta, algunos autores afirman que quizás fue suprimido de su farmocopea por imprecisos estudios de efectos adversos ${ }^{11}$. En España, no obstante, es un medicamento ampliamente utilizado. Así, para hacernos una idea de su empleo, en nuestro centro de salud y en el periodo comprendido entre enero y agosto de 2001 se realizó un consumo de 10.398 envases de analgésicos no narcóticos, de los cuales 2.411 correspondieron al metamizol, lo que supone el 23,19\% del total. Como conclusiones nos gustaría resaltar la importancia de tener presentes los posibles efectos secundarios de aquellos medicamentos que recetamos en nuestras consultas, pues en algunas 
ocasiones pueden ser letales. En vista de los pocos datos de que aún disponemos, no podemos descartar un predisposición genética en los familiares de los pacientes que hayan sufrido agranulocitosis por metamizol, por lo tanto nos parece prudente que no utilicen dicho fármaco mientras no se dispongan de evidencias suficientes de su inocuidad. La dificultad diagnostica de este tipo de cuadros debido a la sintomatología poco específica, hace que debamos tener siempre presentes posibles reacciones medicamentosas. De ocurrir dichos efectos secundarios debemos realizar declaración de los mismos al servicio de farmacovigilancia que corresponda.

\section{CORRESPONDENCIA:}

Israel Sánchez Lite

$\mathrm{C} /$ Puente Colgante, $19, \mathrm{p} 9^{\circ} \mathrm{F}$

47007 Valladolid

Telf.: 983235704

Movil: 605877701

\section{Bibliografía}

1. Márquez JA, Pardo $\mathrm{C}$, Amutio $\mathrm{E}$, Cortés $\mathrm{C}$, Piñán MA, Alvarez C. Agranulocitosis inducida por fármacos: estudio clínico de 19 casos. Sangre 1998; 43 (5): 436-8.

2. Sabbaga J, Isawa C, Pahl FH, Vellutini E, Pereira D, Ceccanello I. Acute agranulocitosis after prolonged high-dose usage of intravenous dipyrone toxicity? Annals of Hematology 1993; 66: 153-55.

3. International agranulocitosis and aplastic anemia study. Risk of agranulocitosis aplastic anemia. A first report of their relation to drug use with special references to analgesics. JAMA 1986; 256: 1749-57.

4. Levy M. Role of Viral Infections in the Induction of Adverse Drug Reactions. Drug Safety 1997; 16 (1): 1-8.

5. Willfort A, Lorber C, Kaprotis S, Sertl S, Hainz R, Kirch Weger $\mathrm{P}$, et al. Treatment of drug induced agranulocitosis with recombinant granulocyte colony stimulating factor (rh GCSF). Ann Hematol 1993; 66: 241-4.
6. Torres Bandía FI, Guevara Serrano J, Carmona Ibáñez G, Ortega García P, López Briz E. Agranulocitosis por metamizol: Descripción de un caso. Atención Farmacéutica 2000; 2 (3): 259-62.

7. Vlahov V, Bacracheva N, Tontcheva D, Naumova E, Mavrudiera M, Ilieva P, et al. Genetic factors and risk of agranulocitosis from metamizol. Pharmacogenetics 1996; 6: 67-72.

8. Vallés i Callol JA. Trastornos hematológicos inducidos por fármacos. FMC 1998: 363-78.

9. Framptom JE, Lee CR, Faulds D. Filgastrim. A review of its pharmacological properties and therapeutic efficacy in neutropenia. Drugs 1994; 48: 731-60.

10. Mani S, Barry M, Concato J. Granulocyte-colony stimulating factor therapy in drug-induced agranulocitosis. Arch intern Med 1993; 153: 2500-1.

11. Cheston Berlin, MD. Are We losing Good Drugs? Clinical Pediatrics 2001; 40: 325-6. 